livraisons

d'Histoire

de l'Architecture

\section{Livraisons de l'histoire de l'architecture}

$23 \mid 2012$

Varia I

\title{
La Place Saint-Michel : Une composition monumentale hiérarchisée du Paris haussmannien
}

The Saint-Michel square: a monumental composition of Haussmannien Paris

Der Platz Saint-Michel: eine besonders ansehnliche Einrichtung im

Hausmannischen Paris

Grégoire Alessandri

\section{OpenEdition}

Journals

Édition électronique

URL : http://journals.openedition.org/lha/134

DOI : $10.4000 /$ /ha. 134

ISSN : 1960-5994

Éditeur

Association Livraisons d'histoire de l'architecture - LHA

Édition imprimée

Date de publication : 15 juin 2012

Pagination : 65-86

ISSN : 1627-4970

\section{Référence électronique}

Grégoire Alessandri, « La Place Saint-Michel : Une composition monumentale hiérarchisée du Paris haussmannien », Livraisons de l'histoire de l'architecture [En ligne], 23 | 2012, mis en ligne le 15 juin 2014, consulté le 01 mai 2019. URL : http://journals.openedition.org//ha/134 ; DOI : 10.4000//ha.134

Ce document a été généré automatiquement le 1 mai 2019.

Tous droits réservés à l'Association LHA 


\title{
La Place Saint-Michel : Une composition monumentale hiérarchisée du Paris haussmannien
}

\author{
The Saint-Michel square: a monumental composition of Haussmannien Paris \\ Der Platz Saint-Michel: eine besonders ansehnliche Einrichtung im \\ Hausmannischen Paris
}

Grégoire Alessandri

1 Boulevards de Sébastopol, du Palais, Saint-Michel : le nouveau cardo de Paris est constitué de plusieurs segments, successivement ouverts du nord au sud, appuyés sur le tissu urbain existant. "Voie de mauvaise volonté ${ }^{1}$ " raille Louis Hautecœur, il forme une chicane place du Châtelet, un arc dans l'île pour joindre les deux ponts, une bifurcation rive gauche où le choix d'orienter le boulevard Saint-Michel sur la Sainte-Chapelle entraîne un désaxement avec le boulevard du Palais. Depuis le pont, l'œil bute sur le murpignon des immeubles situés à l'angle des boulevards Saint-Michel et Saint-André. Cette situation nécessite un aménagement monumental qui souligne et dissimule à la fois le désaxement. Objet scénographique et décoratif répondant aux nécessités de la visée, la fontaine Saint-Michel affirme également la prise de contrôle de l'espace urbain par le régime issu du coup d'État du 2 décembre 1851. Les fonctions politiques qu'elle remplit à l'évidence retiendront ici d'abord l'attention. On verra ensuite combien les formes nouvelles de monumentalité qu'elle met en œuvre font débat. On s'attardera enfin à préciser les rapports hiérarchiques inédits entre bâti vernaculaire et architecture d'exception qu'inaugure la place Saint-Michel.

\section{La fontaine Saint-Michel : triomphe du régime impérial}

2 Au Second Empire, choisir une fontaine monumentale pour orner une place est déjà en soi l'expression d'une éclatante réussite. En 1850, les besoins des Parisiens sont évalués au double des capacités. Directeur du service des Eaux de Paris, Eugène Belgrand triple 
l'approvisionnement. Place Saint-Michel, «[1]es propriétaires [sont] tenus de prendre pour l'usage des constructions à louer un abonnement aux eaux de la ville et de faire à cet effet dans leur maison les dispositions nécessaires ${ }^{2} »$. Corollaire de cette abondance domestique, l'eau peut être gaspillée à profusion dans des fontaines dont la perte de leurs fonctions traditionnelles 皿 hygiénique (assainissement du réseau viaire) et utilitaire (alimentation en eau potable) [50 accentue le rôle monumental.

Par ailleurs, trois événements concourent à faire triompher le nouveau régime en 1856 : le succès de l'Exposition universelle l'année précédente, la naissance du prince impérial, le congrès de Paris qui met un terme à la guerre de Crimée. Le 9 mai, seize membres du sénat avancent l'idée d'une colonne commémorative située à la croisée du boulevard de Sébastopol-Rive-Gauche et de la rue Soufflot " pour perpétuer le souvenir des victoires de l'armée, et celui de la paix conquise sous les auspices et par le génie tutélaire de S.M. Napoléon III $^{3}$ ». L'absence de sources laisse hélas dans l'ombre la préhistoire de la fontaine. Mais l'idée est séduisante qu'elle soit née des décombres d'une colonne utopique.

Sa destination propagandiste et la position ostentatoire qu'elle occupe au cœur du Paris rénové par le Second Empire placent naturellement la fontaine Saint-Michel sous le feu des critiques. C'est à un jeune architecte de 32 ans cependant que le préfet Haussmann en confie l'édification, justifiant son choix par « le beau caractère de sa fontaine du square des Arts-et-Métiers ${ }^{4} »$. Dès ses premières esquisses (ill. 1 et 2), en juin 1856, Gabriel Davioud (1824-1881) associe une place «bordée de constructions uniformes et plantée d'arbres ${ }^{5}$ » à une fontaine et fige l'ensemble dans une posture triomphale. Il multiplie habilement les allusions au Premier Empire : les immeubles reprennent l'élévation de la rue de Rivoli ; l'association de colonnes de marbre rouge et de boucliers visible à la fontaine semble directement inspirée de l'arc du Carrousel. 


\section{1.Vue de face et travée des faces latérales de la place}

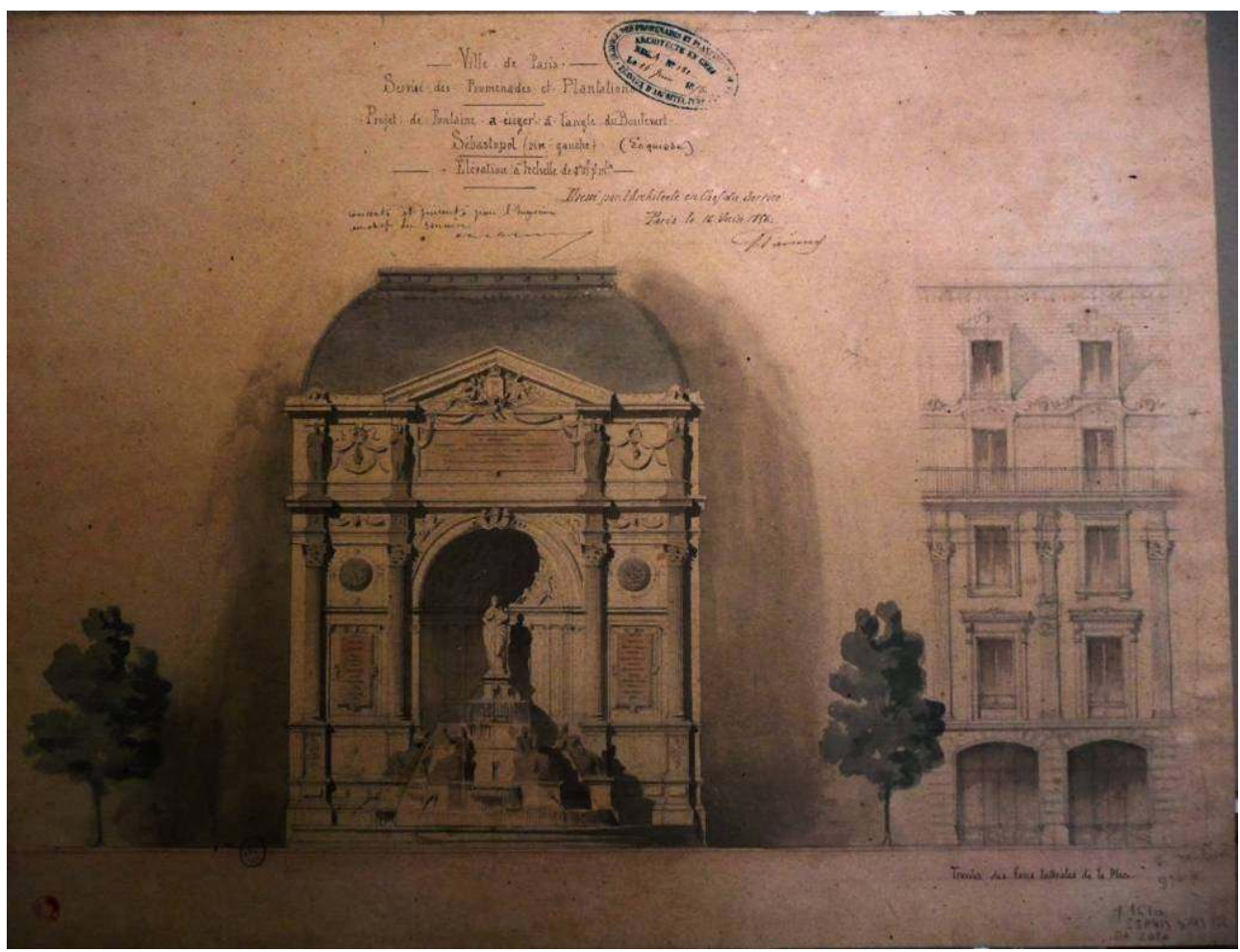

Projet signé du 16 juin 1856. Bavp, fonds Davioud, SP 413 b (1) CR, DA 2057, aquarelle, 34,7 x 45,7 cm. Cl. G. Alessandri 


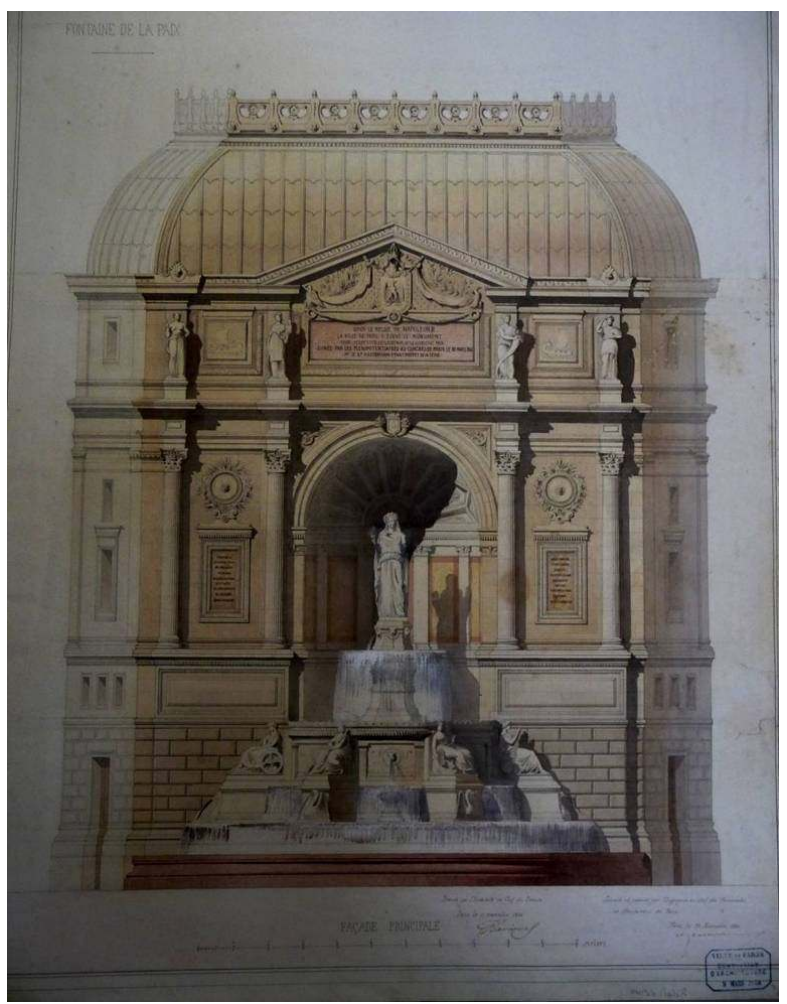

Projet signé du 21 novembre 1856. Bavp, fonds Davioud, SP 413 b (10) CR, DA 2053, aquarelle, 64 x $50,5 \mathrm{~cm}$.

Cl. G. Alessandri

5 À cette architecture de filiation impériale, Davioud surimpose une iconographie elle aussi triomphale. En forte saillie, le groupe central varie souvent: représentation conventionnelle de la Paix successivement chargée d'un enfant, d'une corne ou d'un rameau. L'inscription dédicatoire rappelle que « sous le règne de Napoléon III, la ville de Paris a élevé ce monument pour perpétuer le souvenir de la glorieuse paix signée par les plénipotentiaires au congrès de la paix, le 30 mars $1856^{6}$ ».

Président de la commission consultative des Beaux-Arts de la Ville de Paris qu'il a instituée en 1854, Haussmann désigne, dans une lettre du 10 mars $1858^{7}$ demeurée semble-t-il inédite, le comte de Nieuwerkerke, directeur général des Musées impériaux, le sulpteur Francisque Duret et l'architecte Jacques-Ignace Hittorff, pour faire partie d'une sous-commission chargée d'examiner le projet. Une seconde version (ill. 3), « suivant les indications de M. le préfet et de la commission des Beaux-Arts ${ }^{8}$ ", est présentée par Davioud en avril ${ }^{9}$ et soumise à la délibération du conseil municipal ${ }^{10}$. Le fonds Davioud de la bibliothèque administrative de la Ville de Paris ne permettant de repérer que deux états successifs, on peut penser que c'est l'ambiguïté du dessin qui amènent François Lacour $^{11}$ et Tourettes ${ }^{12}$ à voir une allégorie féminine de la France dans le Napoléon I ${ }^{\text {er }}$ arborant une aigle, que Davioud choisit pour remplacer la Paix. 
III. 3. Projet de "fontaine de la Paix », modifié suivant les indications de M. le préfet de la Seine et de la commission des beaux-arts

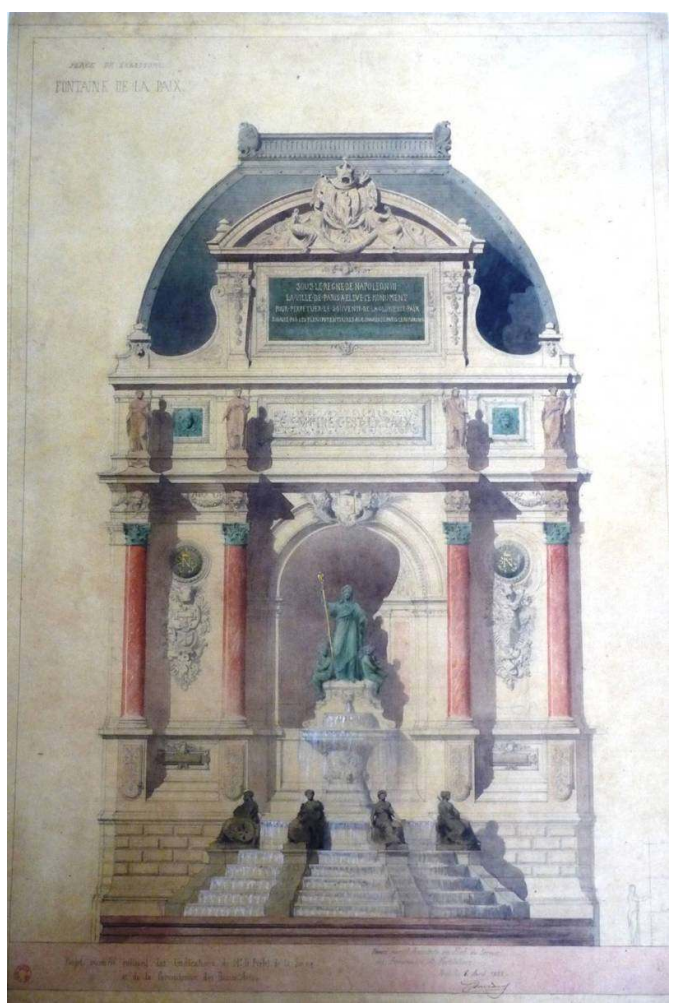

Projet signé du 6 avril 1858. Bavp, fonds Davioud, SP 413 b (12) CR, DA 2054, aquarelle sur calque, $64,3 \times 44 \mathrm{~cm}$.

Cl. G. Alessandri

7 Inspiré de son portrait par François Gérard, la figure de l'empereur est flanquée des génies de la Paix et de la Guerre ${ }^{13}$. Elle est surmontée de la Force, la Sagesse, l'Abondance et la Concorde ${ }^{14}$. À l'attique, une inscription garantit solennellement : «L'Empire, c'est la Paix. ${ }^{15} »$ Ornant les bassins, l'Agriculture, l'Industrie, le Commerce et les Beaux-Arts ${ }^{16}$ personnifient les heureuses conséquences du régime impérial dont Davioud, note Dominique Jarrassé, se fit rarement aussi clairement le chantre ${ }^{17}$. Les rumeurs vont bon train. Camus s'en fait l'écho :

[O]n assure [...] qu'à l'exemple de ce qui a été pratiqué en 1805 pour la colonne de la place Vendôme, le bronze qui servira à fondre les statues de la nouvelle fontaine sera fourni par les canons pris en Crimée ${ }^{18}$.

8 À partir du $1^{\text {er }}$ juin, la fontaine « est élevé[e] sur caves voûtées, construites en meulières et hourdées en ciment de Portland ${ }^{19}$ ». Très vite cependant le projet est une nouvelle fois modifié. Concession à la virulence de l'opposition républicaine ou signe du durcissement du régime après l'attentat d'Orsini et les débuts de la campagne d'Italie ${ }^{20}$, un Saint Michel terrassant le démon, inspiré de Raphaël, est préféré à Napoléon. Les esquisses de cette troisième version ne sont pas datées. Mais le bulletin statistique des travaux terminés ${ }^{21}$ indique que le projet a été présenté par Davioud le $1^{\mathrm{er}}$ avril et le 9 juillet 1858 . La première date correspond à la deuxième version de la fontaine. Il semble raisonnable d'en déduire que la dernière version date du 9 juillet.

9 Une fois achevées les fondations, la construction du monument lui-même débute au mois d'octobre $1858^{22}$ à l'abri derrière un voile « [c]ar on veut ménager au public un plaisir 
imprévu tout en laissant à l'artiste la satisfaction de ne pas livrer à la critique anticipée de la foule un travail inachevé23 » explique Charles Friès. En mai de l'année suivante « [t]out le gros œuvre de la fontaine [...] est pareillement achevé, et l'on va commencer les travaux d'ornementation ${ }^{24} »$. Les quatre colonnes sont placées le 11 avril $1860^{25}$. Le $1^{\text {er }}$ juin débute la construction du rocher ${ }^{26}$. Le 16 juillet deux bassins sont terminés "et les matériaux du troisième sont près d'être mis en place ${ }^{27} »$. Le 29 , les vertus sont hissées sur les colonnes. Le travail s'achève le 8 août 1860 par l'installation dans sa niche du groupe principa ${ }^{28}$. Le 13 , "presque entièrement débarrassée de ses échafaudages ", le monument est visité «par un grand nombre de curieux ${ }^{29}$ ». On s'affaire le 14 à la veille de l'inauguration:

[U]n voile qui tombera à l'heure fixée couvre seulement le groupe de saint Michel et les deux boucliers de bronze qui portent le chiffre impérial [...]. Des mâts vénitiens, surmontés de banderoles tricolores, marquent les abords du monument, où les eaux doivent jaillir en même temps que le pont au Change sera ouvert à la circulation ${ }^{30}$.

10 Le 15 août 1860 , jour anniversaire de la naissance de Napoléon $\mathrm{I}^{\mathrm{er}}$, en présence d'une "foule d'artistes et de curieux ${ }^{31}$ », la fontaine Saint-Michel est inaugurée. Son «luxe architectural inusité32 », relevé par Augustin-Joseph du Pays, et « la belle perspective [...] qu'on [en] a [...] du nouveau pont, en arrivant sur la place ${ }^{33}$ " procurent toute satisfaction à Haussmann. L'appréciation de la plupart des commentateurs est moins positive cependant. Aux yeux d'Alfred Darcel la fontaine semble un «assemblage de statuaire placé au hasard ${ }^{34}$ »; produisant un "pêle-mêle étrange et sans nom ${ }^{35}$ » ajoute Charles Blanc. En 1981, Dominique Jarrassé avance une explication au désarroi des contemporains de Davioud: «[L]a cohérence iconographique et formelle de la fontaine [est] disloquée par le changement du thème central. ${ }^{36}$ " La fontaine se conforme à une "thèse politique et administrative ${ }^{37}$ ", avertit cependant la muse de l'Allégorie surgie opportunément à la rescousse de Jules-Antoine Castagnary dès 1860 . Son "ornementation est conduite à l'effet de mettre en relief l'un des plus grands faits moraux de notre époque: le Socialisme vaincu ou plutôt converti par l'Empire ${ }^{38}$ ». Éclaircie de la sorte, l'idée d'incohérence iconographique paraît peut-être moins soutenable. D'abord parce que, placée dans un arc de triomphe, la victoire du Bien sur le Mal affirme de façon redondante, certes, mais efficace, la vocation triomphale du monument. Ensuite parce que les allégories qui encadraient la Paix étant conservées, l'image de l'ange exterminateur est habilement détournée vers celle d'un ange pacificateur ${ }^{39}$, occupé à convertir le démon en l'invitant à pratiquer des vertus civiques expressément désignées. Devant cette «image philosophique de la vie traçant le devoir de l'homme, comme celui de tout pouvoir protecteur et vigilant ${ }^{40} »$ caressé par Friès, Blanc a beau ironiser : «Ce que l'archange montre du doigt, ce n'est pas l'emblème du paradis, c'est l'écusson de l'Empire ${ }^{41}$ ", on passe volontairement de l'idée d'une Paix statique, triomphante et augustéenne à celle d'une Pacification en action. La muse de Castagnary précise encore le propos :

Dans le bas, les énormes Chimères, c'est-à-dire les faux systèmes, les mauvaises doctrines, les erreurs détestables nées sous la République de 1848, sont assises sur leur croupes, impuissantes désormais ${ }^{42}$.

11 La guerre franco-prussienne de 1870 sert d'épilogue brutal à cette ère polémique, mais paisible. La Commune prend le trop allégorique monument, pacifique ou pacifiant qu'importe, pour cible. Davioud s'en inquiète dans une lettre au directeur des Promenades et Plantations :

Une troupe d'ouvrier sans armes vient de se présenter à la fontaine Saint-Michel et s'occupe de force à enlever les aigles et chiffres impériaux qui étaient placés dans le 
vestibule. Ils vont évidemment saccager la fontaine et vouloir enlever les aigles et chiffres qui ornent les parties supérieures. Que dois-je faire ${ }^{43}$ ? de ses détracteurs, qu'il s'agisse de dissimuler l'articulation défaillante de deux boulevards ou d'exprimer triomphalement les réussites du régime, l'option retenue multiplie les obstacles à l'effet recherché. Or, loin de se limiter à la condamnation des maladresses d'un débutant, la virulence des attaques et ce qu'on devine des réticences de Davioud lui-même dressent le tableau d'une époque troublée par les options nouvelles du «monumental». C'est en effet une monumentalité inédite qui est instaurée place SaintMichel, et par de nouveaux moyens que s'y exprime la relation hiérarchique inévitable entre constructions vernaculaires et bâtiment d'exception. dont elle souffre par rapport au pont contredit l'idée que se fait Darcel de la "monumentalité »: «Il aurait fallu la soulever à une certaine hauteur par un socle [...] rachetant la pente du terrain [...]. Au lieu de cela tout le soubassement de la fontaine, jusqu'à la naissance de la niche, avec sa série de vasques descendant jusqu'au sol, est caché pour quiconque arrive par le pont ${ }^{46}$.» compense cette position défavorable par une composition très élevée (26 sur 15 mètres) L'eau tombe de près de 7 mètres. À l'origine «entouré d'une plate-bande de gazon et d'une petite grille ouvragée ${ }^{48}$ ", le grand bassin placé à ras du sol et les quatre vasques superposées isolent le monument à la façon d'un soubassement et le lancent à l'ascension du mur-pignon. Davioud apporte ici une réponse crédible à la question du socle qui taraude le siècle et témoigne de la crise du monumental qu'il traverse. forme retenue n'est pas une innovation pourtant. Elle se démarque des précédents

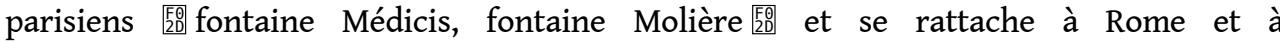
l'enseignement néo-classique des Beaux-Arts. Mais la critique juge sévèrement ce choix. Elle en attribue la responsabilité à la commission des Beaux-Arts de la ville, présidée par Haussmann, qui imposerait à Davioud, «mis au supplice ${ }^{49}$ " à en croire Fournel, la disposition très contraignante d'un monument adossé. Dans l'hommage posthume et donc nécessairement un peu hagiographique qu'il lui rend, Denis-Louis Destors dégage l'artiste de la responsabilité d'un parti maladroit «imposé par l'administration»: «Davioud ne voulait pas incorporer la fontaine dans l'ordonnance des maisons de la place. Il avait conçu un monument détaché ou du moins en avant du pan coupé ${ }^{50}$. »

17 Il n'est pas impossible que, dans ce «ou du moins» équivoque, se dissimule le pieux mensonge d'une profession qui fait corps derrière un confrère. Il nous est difficile, en effet, d'accréditer l'idée selon laquelle Davioud aurait d'abord songé à « un monument au centre de la place, indépendant de l'ordonnance des maisons qui font face au pont» 
comme le conjecture Dominique Jarrassés ${ }^{51}$. L'imprécision de Rossigneux évoquant la place et la fontaine " qui devait en occuper le centre ${ }^{52}$ " n'emporte pas notre conviction. Au contraire, tous les projets attestés par le fonds Davioud concernent une fontaine adossée strictement intégrée aux immeubles voisins. Dès le départ, la modestie de sa situation est très volontairement choisie. Charles Friès en témoigne dès 1859 :

Une profondeur de 5 mètres [...] ayant été réservée, le surplus du terrain a été vendu [...]. Les travaux de la maison et du monument ont été entrepris simultanément au commencement de l'été dernier et poursuivis sans interruption ${ }^{53}$

Précisément, un troisième reproche adressé à Davioud concerne le rapport de promiscuité qu'entretient le monument avec les habitations qui l'entourent. «Situation peu judicieuse $\mathrm{e}^{54}$ », déjà, pour les plus modérés, l'apposition de la fontaine aux immeubles prend, chez Augustin Joseph du Pays, le ton louche d'un "système essentiellement vicieux ${ }^{55}$ » ou même les sinistres accents d'une «erreur capitale ${ }^{56}$ ». Les précédents ne manquent pourtant pas. À la fontaine Gaillon, en 1828, Louis Visconti reprend la liaison visuelle du monument et de son environnement expérimentée par Nicola Salvi à la fontaine de Trevi dès 1732. Mais la critique refuse sévèrement à Davioud le droit d'adosser son œuvre à cette solide tradition. La covisibilité du monument avec des " constructions cruellement bourgeoises ${ }^{57}$ ", pour reprendre le mot de Louis Lazare, " gêne nécessairement l'œil ${ }^{58}$ " s'insurge Castagnary. Si le talent de Bouchardon évite une promiscuité infamante à la fontaine des Quatre-Saisons « au moyen du plan circulaire de sa façade, qui l'isole [...] des bâtiments qui l'accompagnent ${ }^{59}$ ", Félix Narjoux reproche à l'inverse à Davioud "d'épouser les principales lignes de raccord des bandeaux et corniches des maisons voisines ${ }^{60}$ » et Augustin Joseph du Pays regrette "une triste solidarité entre l'œuvre de l'artiste et la construction du bâtisseur ${ }^{61}{ }^{\prime}$. Derrière la stupeur se devine une redéfinition du rapport hiérarchique monumental traditionnel. La densité de l'argument oblige à l'aborder en deux temps: la fontaine d'abord, les immeubles ensuite.

\section{Une hiérarchie monumentale renouvelée : la fontaine}

La fontaine Saint-Michel trouble les habitudes de son époque. Dans le système classique, affirme François Loyer, «le monument est fondamentalement «objet»: isolé, hétérogène, autonome et autocentré ${ }^{2} »$, tranchant vivement sur l'échelle du vernaculaire. Avec la ville monumentalisée que propose l'haussmannisation cette opposition traditionnelle s'atténue au point de disparaitre alors que des distinctions hiérarchiques nouvelles s'établissent entre bâtiments signalant l'exception eux-mêmes. Le hors-d'échelle, quadrifront et isolé, est désormais limité au degré le plus haut de la gamme monumentale. À l'échelon immédiatement inférieur le monument est soumis au réseau mais continue à le dominer malgré tout par un jeu de combles immenses et par l'écriture contrastée des détails. Au degré le plus bas, il range sa façade à l'alignement des immeubles, le volume du comble faiblit et n'est plus marqué que par quelques crêtes découpées. L'architecture seule distingue le monument ${ }^{63}$.

Place Saint-Michel, l'évolution est largement accomplie. Le monument se glisse dans le triangle laissé vide par la bifurcation de la voirie. Cette situation trop modeste lui est reprochée. Placé dans une situation très similaire, le dôme de Saint-Augustin ${ }^{64}$ parvient cependant à imposer sa forte silhouette à un environnement disparate. Mais notre 
fontaine à elle seule n'a pas le poids nécessaire pour structurer, stabiliser, bref "précipiter" visuellement un carrefour en place. Sa timidité relative et l'échelle majestueuse des immeubles brouillent les distinctions traditionnelles du monumental. Dominique Jarrassé insiste sur le caractère volontairement novateur de cette solidarité. Composant en même temps la fontaine et les façades obligatoires des immeubles Davioud prend grand soin d'imposer à l'ensemble un décor et un rythme communs. Le soubassement de la fontaine correspond au rez-de-chaussée des immeubles, les colonnes à l'ordre colossal de pilastres, l'attique au balcon filant. Le rigoureux dessin de Davioud conduit le regard à suivre «certaines lignes de force horizontales focalisées sur la fontaine ${ }^{65}$ ». Les «impertinentes concordances de hauteurs ${ }^{66}$ », regrettées par Pays en 1860 , deviennent « jeu de correspondances ${ }^{67} »$ chez Dominique Jarrassé et définissent la place en tant qu'objet formel homogène. On devine toutefois qu'un tel système prend le risque de dissoudre le monument dans une ligne d'horizon inarticulée. La nécessité s'impose alors d'inventer des critères nouveaux qui permettent de "re-connaître » la monumentalité. Il s'agit essentiellement d'une polychromie exubérante et de l'abondance du décor sculpté.

\section{La polychromie}

21 Davioud manifeste très tôt un vif intérêt pour la couleur. En 1845 déjà, il anime l'esquisse d'arc qu'il rend au concours d'émulation des Beaux-Arts de marbres rouges et verts ${ }^{68}$. Si les premiers dessins de la fontaine Saint-Michel font un usage prudent de la polychromie, celle-ci prend ses aises en revanche, joyeuse et irrépressible, à la surface du projet définitif. Quelle part prennent les membres de la commission des Beaux-Arts de la Ville à cette inflexion? La destruction des archives conservées à l'Hôtel de Ville empêche hélas d'en juger mais il n'est peut-être pas déraisonnable de croire que furent décisives les préférences d'Hittorff. Quoi qu'il en soit, dans le débat sur la polychromie qui agite les architectes de l'époque ${ }^{69}$, Jules Bourdais souligne combien la fontaine Saint-Michel constitue une «tentative absolument nouvelle [...] de l'emploi de marbres colorés dans la décoration extérieure des monuments de Paris $^{70}$ ».

Il s'agit en premier lieu, stipule la notice officielle ${ }^{71}$, de compenser le défaut d'ensoleillement dû à l'orientation défavorable du monument par la coloration de ses volumes même. Ce moyen permet également de distinguer la fontaine des immeubles monochromes qui l'entourent. Les vasques et le bassin sont en pierre de Saint-Ylie (Jura), d'un gris jaune nuancé de rouge; le rocher de l'archange en pierre bleue de Soignies (Belgique); le reste de l'élévation en pierre blanche de banc royal de Méry (Oise). Les marbres sont aussi utilisés en abondance : rouge, blanc ou vert. Enfin Davioud joue des patines du bronze : claire pour l'archange, plus foncée pour le diable. Mais, à l'inverse du but poursuivi, l'opinion décontenancée considère majoritairement que cet «essai d'architecture polychrome ${ }^{72}$ » fait perdre au monument «de sa prestance par le papillotage des couleurs ${ }^{73}$ » et nuit à sa position dominante. Victor Fournel est d'une extrême sévérité : "On a voulu suppléer à la richesse de la conception par celle de l'exécution, en poursuivant la variété par l'emploi hasardeux des matériaux multicolores ; on n'est arrivé qu'à la bariolure ${ }^{74}$. »

23 L'indignation publique atteint son comble à la vue des trois motifs géométriques du sommet, un cercle qu'entourent deux carrés composés de marbres colorés (ill. 4). 


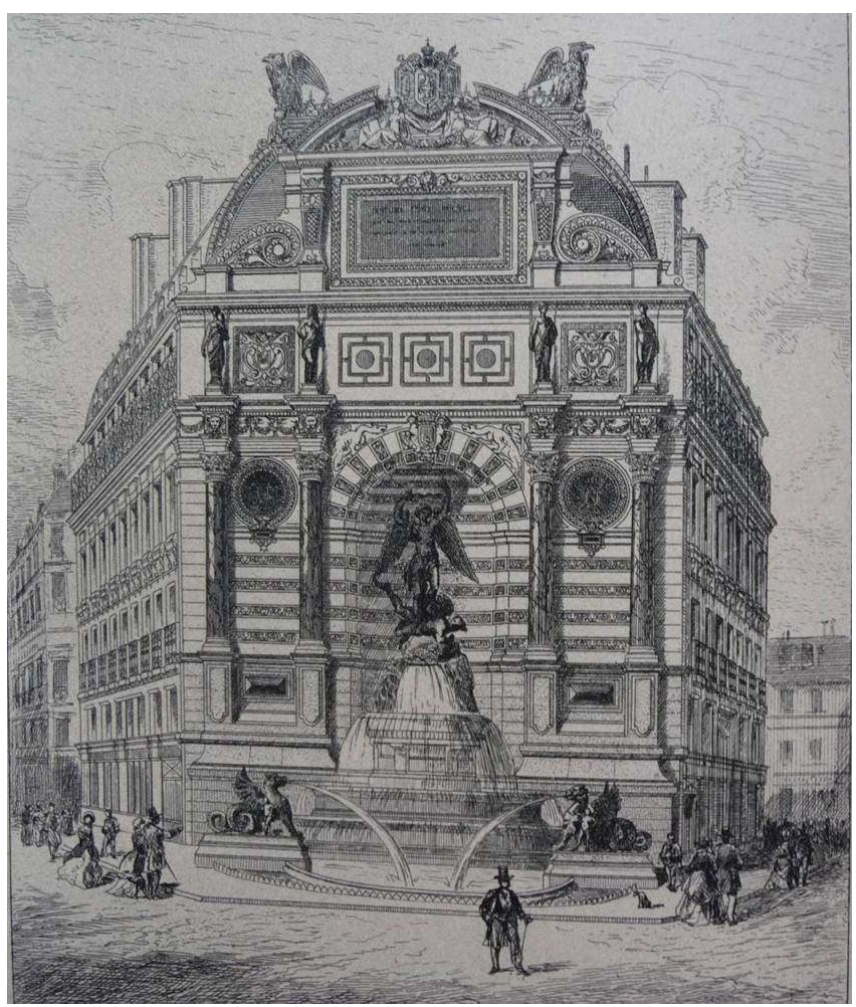

Planche gravée, Charles Blanc, « La fontaine Saint-Michel », dans Gazette des beaux-arts. Courrier européen de l'art et de la curiosité, octobre 1860, tome VIII, p. 51-55, p. 54.

Haussmann ne s'intéresse guère à la polychromie ${ }^{75}$. Davioud doit céder à l'opinion. En 1863, les rinceaux et les enfants de la sculptrice Noémie Constant que nous voyons aujourd'hui sont posés ${ }^{76}$. La couleur, admise théoriquement quand elle est restituée aux belles élévations antiques que dessinent les élèves des Beaux-Arts, suscite l'hostilité lorsqu'elle est appliquée en pratique aux monuments publics. Mais Dominique Jarrassé rend justice à Davioud d'avoir exploré «une possibilité de renouveler les thèmes classiques et de diversifier le paysage urbain ${ }^{77} »$.

\section{Le décor sculpté}

Un décor abondant et varié constitue le second moyen mis en œuvre par Davioud pour assurer à sa fontaine une position dominante sur son environnement. Dominique Jarrassé s'attend naturellement à voir "l'eau, élément essentiel de mouvement ${ }^{78}$ ", occuper une place majeure dans cette grande composition ornementale. Or « [c] urieusement, s'étonne Béatrice Lamoitier, à aucun moment n'est évoquée [cette] question ${ }^{79}$ " chez les contemporains de Davioud. À peine apprend-on que les travaux de fontainerie sont exécutés par Halo sous la direction de Belgrand ${ }^{80}$ ou que le débit atteint « 23 litres par seconde $^{81}{ }$. Aucun commentaire, dépréciatif ou élogieux, n'adoucit cette sèche précision technique. L'invraisemblance du dispositif hydraulique frappe négativement il est vrai, l'eau paraissant sourdre d'habitations, mais le débat se concentre essentiellement sur le décor. Bien plus que le manque d'eau c'est le manque d'impression d'eau qui choque à l'inauguration. L'élément liquide importe moins que sa transcription architecturale qui doit exprimer clairement la fonction du bâtiment. Augustin Joseph du Pays donne la 
recette : «[P]our l'ornementation d'une fontaine monumentale [...] les naïades, [...] les dauphins, voire même la vieille urne classique, restent toujours les motifs les plus gracieux $^{82}$.»

Sans doute la discrétion de l'élément liquide est-elle relative. La polychromie, on l'a vu, mais aussi l'agitation décorative éclipsent tous les jeux d'eau. Au centre de la composition un saint Michel de bronze terrasse le démon dans une niche ornée de bossages. Les colonnes qui entourent cette effigie sont prolongées par les statues des vertus cardinales. Dans leurs intervalle sont disposés des boucliers portant un « $\mathrm{N}$ » sommé de la couronne impériale ${ }^{83}$. Le cartouche au-dessous est orné d'une tête d'ange. Plus haut, la frise de l'entablement est sculptée de putti tenant des guirlandes. Un écusson à tête de lion orne le droit de chaque colonne. Les bas-reliefs situés entre les vertus réitèrent la lutte de l'archange et du diable : des angelots affrontent des dragons dans des rinceaux d'acanthe. L'ensemble est surmonté à l'attique d'une inscription commémorative: «Fontaine de Saint-Michel. Sous le règne de Napoléon III, empereur des Français, ce monument a été élevé par la Ville de Paris, l'an MDCCCLX. » Ce panneau est cantonné de pilastres gainés et surmonté d'un masque entouré de volutes végétales. Encore plus haut, le fronton brisé de la fontaine est frappé des armoiries de l'empereur (aujourd'hui l'écu à casque de la Ville de Paris), avec sceptre et main de justice qu'encadrent la Puissance et la Modération. « La superposition culmine avec deux aigles impériales [noires, en plomb repoussé au marteau] aux angles du toit bombé ${ }^{84} »$ couvert en ardoise.

Accroupies sur des piédestaux en avant du bassin, les deux grandes chimères domptées par les petits génies «accompagnent merveilleusement le motif principal, certifie la notice officielle, et y conduisent l'œil sans effort, tout en préparant la pensée à l'idée qu'il éveille à l'esprit ${ }^{85}$ ». À l'inauguration, l'exotisme des modèles de plâtre déchaîne pourtant les sarcasmes. Les enfants, raillés, « manqu[a]nt absolument de caractère et de style ${ }^{86}$ » au dire de Charles Blanc, sont supprimés en $1861^{87}$, quand sont fondues et mises en place les sculptures définitives sur des piédestaux surélevés ${ }^{88}$.

Là ne s'arrêtent cependant pas les critiques. Les guides Joanne reprochent à la composition d'ensemble son "manque de relief, [s]a maigreur ridicule ${ }^{89}$ » et le manque d'harmonie de ses parties. Elle évoque, pour Fournel, «ces couvertures en papier gaufré, si fameuses dans les distributions de prix des écoles primaires ${ }^{90} »$. La plupart des sculptures suscite des commentaires acides. Le Temps voit, dans le groupe principal, « un monsieur couché sur le ventre, cherchant à découvrir une fuite d'eau, tandis qu'un autre lui fait la mauvaise farce de lui marcher sur le dos $^{91} »$. Castagnary s'amuse d'un " archange qui, dans la singulière attitude que l'artiste lui a donnée, semble moins disposé à frapper Satan qu'à se couper le bras gauche ${ }^{92}$ » et glisse, venimeux :

«M. Duret [...] s'est montré merveilleux dans les parties de son œuvre où il s'est borné à copier [...] Raphaël; mais, dans celles [...] qui lui sont propres, il est tombé au-dessous du médiocre ${ }^{93}$.»

La philippique n'est pas justifiée par des maladresses techniques. Lance admet sans réticence qu' « [o]n ne pouvait guère mieux tailler la pierre, polir le marbre et couler le bronze » mais déplore «la prédominance du métier sur l'art ${ }^{94}$ ». Plus encore que telle ou telle partie sculptée, la profusion décorative dont témoigne la fontaine Saint-Michel concentre les inquiétudes stylistiques d'une "époque qui ne s'aimait pas ${ }^{95}$ " pour reprendre la formule récapitulative de Dominique Jarrassé. Darcel dénonce la surabondance pompeuse d'un «immense bas-relief, imitation grotesque de quelque tableau ${ }^{96} »$. Louis Lazare condamne "les imperfections de cette composition vulgaire ${ }^{97}$ » 
et Fournel conclut, lapidaire : «Incohérence, c'est le nom de la fontaine Saint-Michel [...]. Autant de parties, autant de styles ${ }^{98}$. "

On ne saurait cependant limiter la fontaine Saint-Michel au «collage », postmoderne avant la lettre, de motifs renaissants. Tentative brillante de renouveler la tradition de la fontaine adossée, elle constitue également une réponse à la crise de la monumentalité dont le $\mathrm{XIX}^{\mathrm{e}}$ siècle est victime. Volontairement Davioud transplante des motifs anciens et surenchérit dans l'abondance. Contraint à l'alignement qui le prive du hors d'échelle et au placage qui lui interdit l'isolement, il affirme la singularité du monument par la complexité volumétrique de ses masses, en opposition à la planéité graphique indéfiniment répétée des façades qui l'environnent ${ }^{99}$. Dès lors, au même titre que la polychromie, la richesse proliférante de l'ornementation doit être considérée comme un moyen et non un but. Elle assure au monument une forte individualité qui en évite la dilution dans l'ampleur inédite des perspectives. La fontaine s'inscrit, mais en rupture, dans la procession rigide des immeubles. On s'arrête pour la regarder de face ; ils se voient de profil « en passant » (ill. 5).

III. 5. Le boulevard et la fontaine Saint-Michel vers 1865

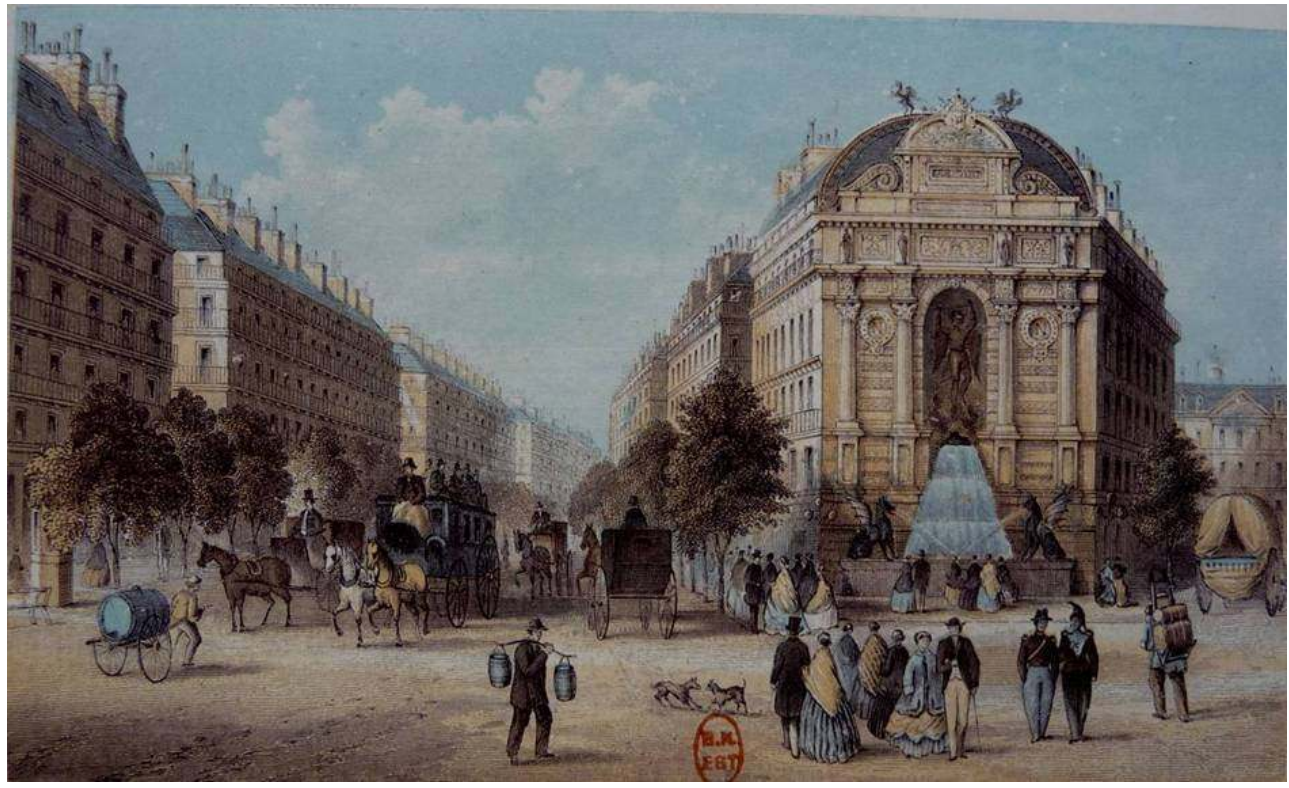

BNF, CE, recueil topographique, Paris, $\mathrm{Vl}^{\mathrm{e}}, 21^{\mathrm{e}}$ quartier, boulevard Saint Michel, Va 263 (G) Fol, image H 44839.

Cl. G. Alessandri

\section{Une hiérarchie monumentale renouvelée : les immeubles}

Contrairement à la légende, aucun dispositif réglementaire n'impose autoritairement aux immeubles haussmanniens des façades rigoureusement répétées. Le décret du 27 juillet 1859 reprend en grande partie les règles fixées sous Louis XVI et, dans le rapport complexe des forces sociales qui fabriquent la ville, les autorités doivent composer avec des propriétaires par principe réticents à la contrainte. La tendance va plutôt à une harmonisation librement consentie, encadrée par la circulaire qu'Haussmann adresse aux 
architectes-voyers en $1855^{100}$. Limitée à quelques espaces prestigieux, le caractère ordonnancé de la place Saint-Michel souligne son rôle éminent dans la ville et s'impose contractuellement aux lotisseurs :

La vente dont il s'agit est encore faite aux conditions suivantes [...] : 1 . D'élever sur le dit terrain une seule maison [...] conformément au système d'architecture adopté pour les constructions de la place du Pont-Saint-Michel. Un exemplaire gravé des plans, coupes, élévations et profils cotés des façades [...] sera enregistré en même temps que les présentes [conditions particulières] ${ }^{101}$. » spécifie [...] la hauteur des étages, des planchers et des allèges de fenêtres; elle donne la profondeur des balcons et des corniches. La façade est encore plus détaillée, avec ses cotes qui précisent, pour les fenêtres par exemple, l'épaisseur des montants, des battants, et des dormants ${ }^{102}$."

Le dessin de Davioud doit s'adapter à la situation nouvelle de l'immeuble dans la ville moderne. La priorité donnée au mouvement et une volonté d'harmonisation globale conduisent à privilégier la vision en ligne de fuite. Ce changement de perspective fait basculer la façade d'une composition axialisée «classique » à une composition profilée marquée par les balcons filants et l'inclinaison des combles. Le décor est saillant sur la façade pour être vu de profil et privé de caractéristiques individuelles trop marquées pour ne pas perturber l'unité d'un ensemble perceptible désormais à l'échelle de la ville. La cohérence de l'ordonnancement urbain détruit l'immeuble en tant qu'objet architectural. Structurellement privés d'autonomie, les immeubles relèvent de la voie désormais et non plus de la parcelle. Au débouché du pont Saint-Michel cependant, le vide immense créé par la place et la perspective sans fin du boulevard exigent d'être stabilisés par des formes d'un poids suffisant. Cette volonté monumentale contredit les exigences circulatoires de la ville moderne. Davioud résout cette contradiction en s'inspirant de grandes ordonnances classiques. Elles lui permettent à la fois d'insérer la place dans la continuité du boulevard, de subordonner les immeubles à la fontaine et de faire jouer à ces derniers une partition autonome. Le vocabulaire décoratif est tiré de la Renaissance, l'élévation de la rue de Rivoli, l'ordre colossal de la place Vendôme. Issu de l'architecture palladienne, le grand comble évoque l'ancien opéra de Debret. Il ne nous semble pas, toutefois, qu'il faille abuser d'une lecture «à clefs ». Dans ce qui relève d'une culture générale de l'architecture ${ }^{103}$, Davioud dissout et recompose les formules historiques à l'aune de son talent propre.

Cette synthèse éclectique fait dialoguer en permanence des perceptions alternées en fonction de la distance. En vision de près, la finesse du détail décoratif dénote la volonté d'égaler la tradition classique dans un contexte marqué par l'excellence de la formation des ornemanistes ${ }^{104}$. Pris individuellement, chaque élément sculpté relève d'une vision axialisée, disons «baroque ». La saillie du balcon et du dais à consoles qui encadre la grande fenêtre constitue ainsi un motif fermement particularisé qui engage d'autant plus à une appréciation frontale que, comme aux façades latérales du Châtelet par exemple, le balcon n'est pas continu. 
III. 6. Système d'architecture obligatoire pour la place Saint-Michel. Élévation et profil cotés des façades

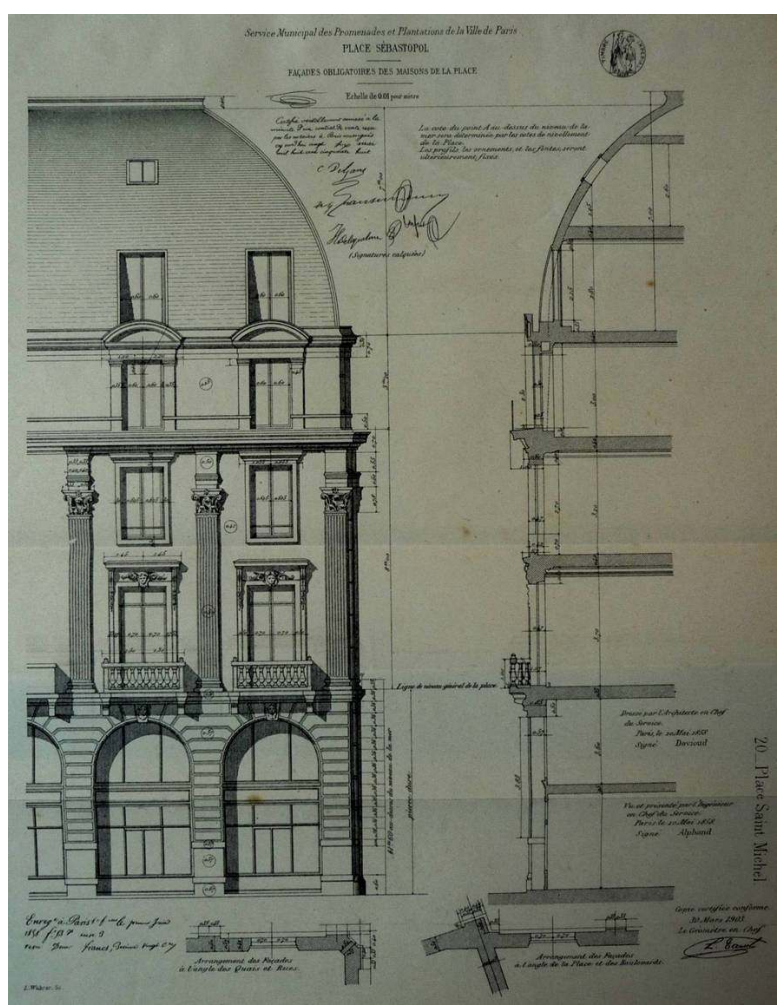

Louis-Charles Taxil, Joseph-Antoine Bouvard (dir.), Recueil d'actes administratifs et de conventions relatifs aux servitudes spéciales d'architecture, aux servitudes non-ædificandi et autres grevant les immeubles riverains de certaines places ou voies publiques, Paris, Imprimerie nouvelle (association ouvrière), 1905, 32 p., p. 23 et planche

Cl. G. Alessandri

Mais ces motifs s'intègrent aussi dans des combinaisons inédites. À une échelle moyenne de perception les verticales dominent. Placés aux clefs des arcs de l'entresol, les mascarons aident les consoles latérales à soutenir le balcon du piano nobile. Cette fusion, en un "dais-appui ", des fonctions de la console et de la clef, lie verticalement les deux premiers niveaux de l'immeuble. La travée ainsi nettement définie est encore accentuée par la réduction progressive de la largeur des baies empilées verticalement jusqu'au toit qui produit l'impression d'une fuite vers le haut. À l'échelle de la masse, Davioud organise ses immeubles en volumes empilés : deux niveaux commerçants inclus dans une arcade, trois étages carrés de hauteur décroissante, un comble sphérique pour couronnement. En vision de loin, les grandes horizontales 㡠 les balcons filants du troisième par exemple mais essentiellement le haut comble 50 医 stabilisent les formes, «tiennent ensemble » les immeubles et donnent son unité à la place. Enfin, à l'inverse de la composition syncopée, normale à l'époque, qui consiste à signaler le grand salon par deux ou trois baies regroupées au centre de la façade, Davioud distribue, place Saint-Michel, une travée d'ordonnance unique sur un rythme parfaitement étale. Encore décelable dans les souches des cheminées, la cadence des mitoyens disparaît par ailleurs et fait oublier l'individualité des immeubles au profit de la majesté de l'ensemble.

En façade s'exercent donc des pressions antagonistes: le détail ornemental pousse l'immeuble vers la frontalité et l'autonomie tandis que les travées répétées et les grandes 
horizontales en refusent au contraire l'axialité et donc l'individualisation. Tout autour de la place en effet, les amples motifs verticaux des percements sont traversés horizontalement par les lignes filantes des corniches et des balcons. Il en résulte un quadrillage ornemental qui, tendu à la façon d'une vaste toile pelliculée à la périphérie du vide central, accentue l'unité à grande échelle de la place.

Étalés en surface, les ornements s'organisent aussi dans la profondeur. La somptuosité de la pierre est mise en valeur par d'amples nus muraux. Mais l'insistance décorative portée aux encadrements révèle une ossature qui défait la matérialité du mur. Sur l'épiderme ainsi rendu diaphane, Davioud dépose une couche de reliefs légers: bossage du soubassement ou chapiteaux corinthiens couronnant les fûts cannelés des étages nobles. À cette première épaisseur, il ajoute le balcon à balustres de la fenêtre du premier étage. Plus avant, sur la surface de pierre que la lumière anime et fait vibrer, se projette en contraste le noir des motifs en fonte du balcon du troisième. Enfin, au contraire de Garnier qui impose de haute lutte la minéralité sévère de l'avenue de l'Opéra, Davioud, architecte du service des Promenades et Plantations, joue volontiers des grands arbres

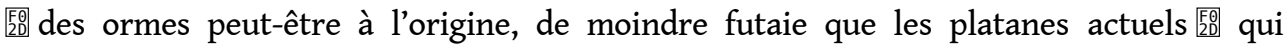
ajoutent l'alternance des saisons à l'impression du lieu. L'été, l'écran formé par leur feuillage oblige à venir au plus près des immeubles pour en apprécier la prolixité des détails. L'hiver, leurs branches dénudées jouent en contrepoint sur la basse continue des façades et leurs troncs forment comme des colonnes détachées à l'avant des pilastres de l'ordre colossal. Ce travail sur la profondeur reste très maîtrisé cependant: les volumes sont comme introvertis, les reliefs ciselés plus que sculptés. Escamotables, les persiennes ne troublent pas ce désir retenu de monumentalité.

Par ces critères d'appréciation renouvelés du monumental, la fontaine s'affirme comme « un accident dans le déroulement continu des façades ${ }^{105}$ ». Son caractère unique joue en contraste sur la pluralité des immeubles, sert de cible à leur alignement et de signal dans la ville (ill. 7). 
III. 7. La place Saint-Michel vue du boulevard du Palais dans les années vingt

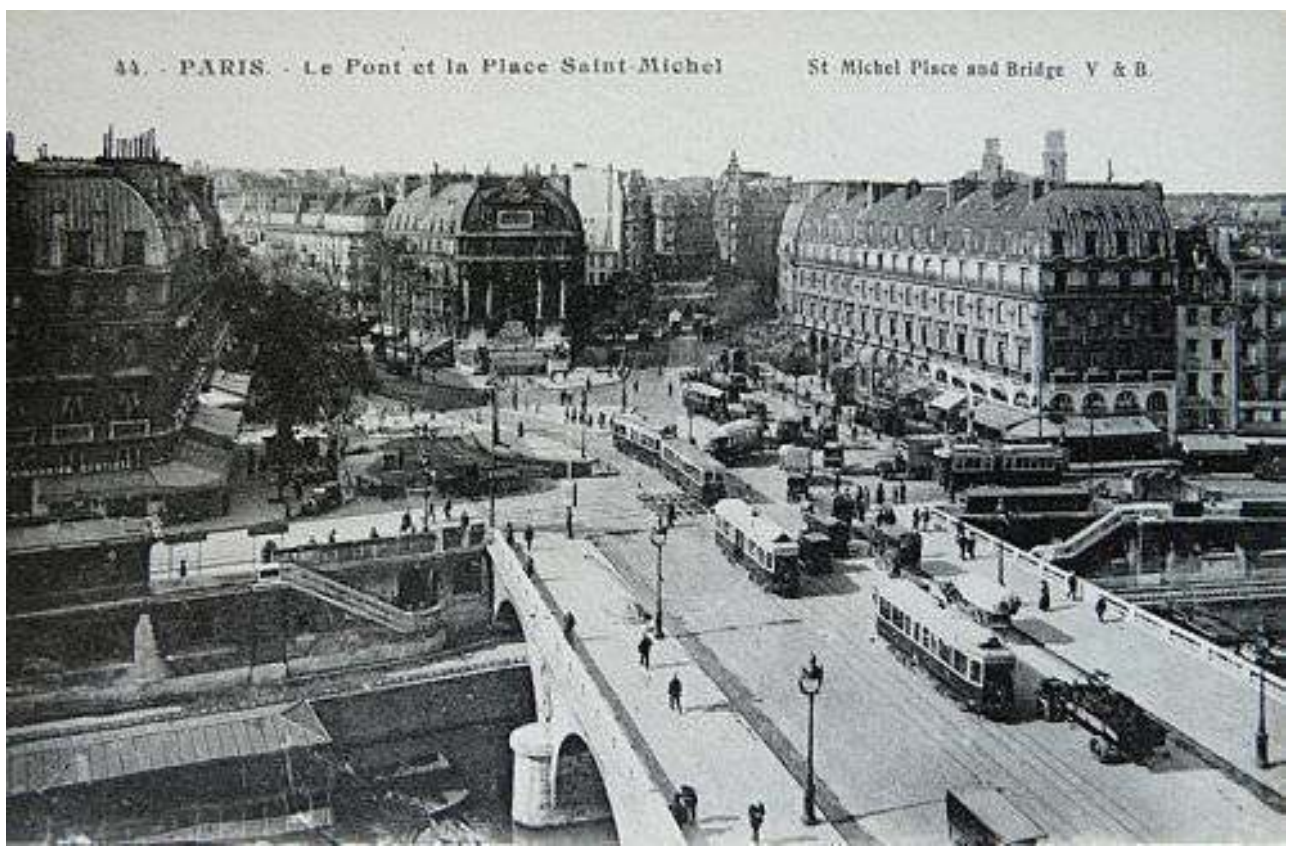

BNF, CE, Topographie de la France. Cartes postales. Paris VIe. CP 780, Va matières 50 (D).

Cl. G. Alessandri

Placé dans la nécessité de compenser le manque d'eau et de soleil, soumis à des contraintes brutales de gabarit, Davioud parvient à créer une forme mouvementée, structurée et originale qui répond parfaitement aux objectifs qui lui sont assignés. À ce titre, et en dépit de programmes incomparables, la vaste scénographie de l'Opéra se déploie de façon très similaire au terme d'une perspective dont le départ est encadré par des immeubles jumeaux (ill. 8). 


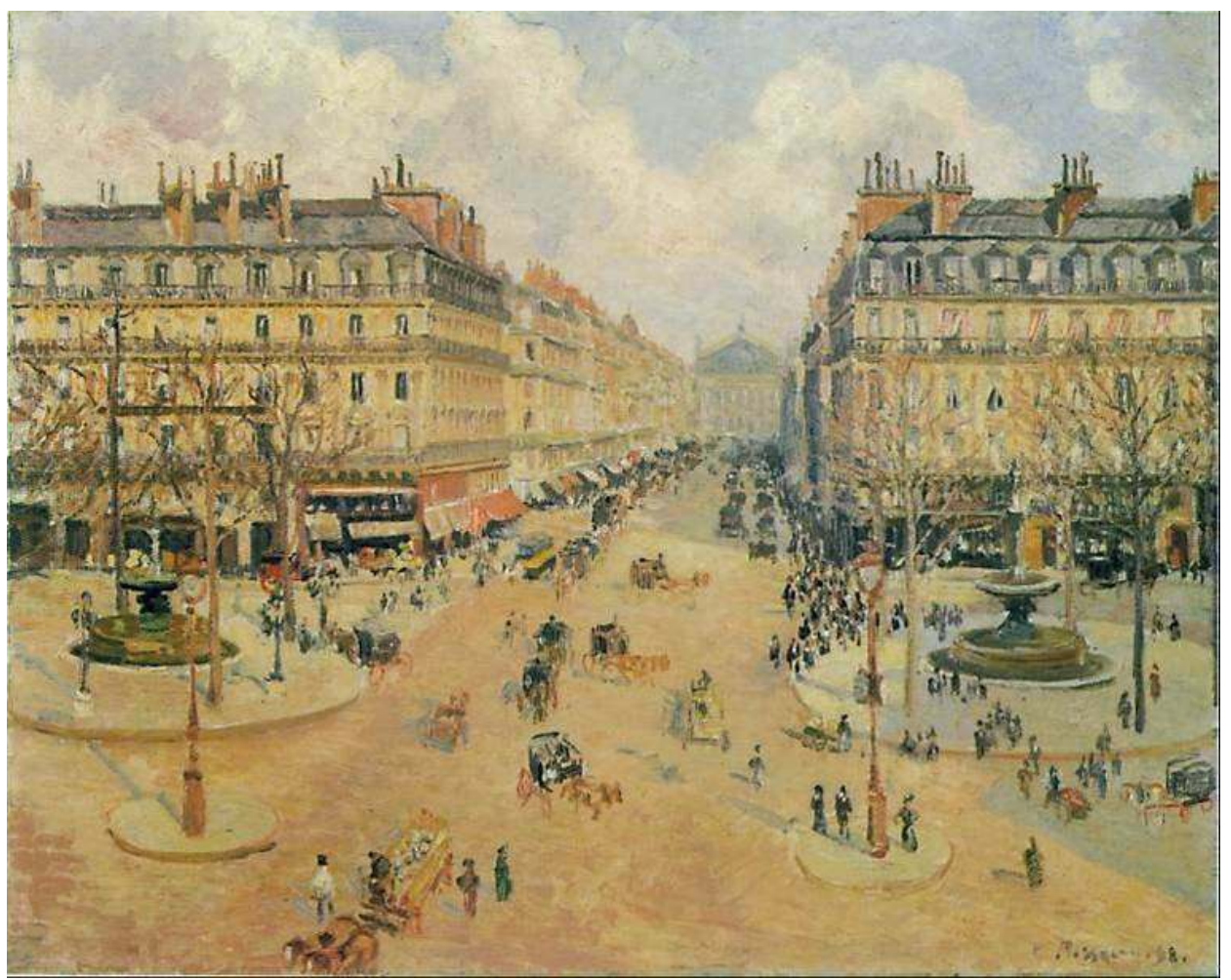

Huile sur toile, 1898, 65 × 81 cm, collection privée, Philadelphie.

Cl. G. Alessandri

Subi par Charles Garnier, désiré au contraire par Davioud, le même contraste formel oppose le monument aux immeubles voisins : relief, exubérance, vive polychromie, d'une part, planéité, austérité, camaïeu, de l'autre. Certes, la fusion ostentatoire des arts, supérieurement pratiquée à l'Opéra, en fait un monument-phare de l'éclectisme impérial là où la fontaine semble souffrir d'une certaine maigreur monumentale. Mais Davioud, comme Garnier, tient brillamment l'impossible pari de différencier le monument et de l'insérer à la fois dans son environnement. Abondante mais ordonnée, éclectique mais rationnelle, la belle calligraphie décorative qu'il déploie résout avec aisance, et presque jubilation, le grave problème formel né de l'opposition des fonctions circulatoire et monumentale de la place Saint-Michel. Tout compte fait, nouveau centre d'un quartier rénové, l'exubérance joyeuse de la fontaine et le treillis austère qui corsète les immeubles affirment en complémentarité l'organisation hiérarchique de l'espace urbain.

\section{NOTES}

1. Louis Hautecœur, Histoire de l'architecture classique en France, t. VII, «La fin de l'architecture classique 1848-1900 », Paris, A. et J. Picard et c Ce 1957, p. 24. 
2. «Conditions particulières imposées par la ville de Paris aux acquéreurs des terrains en façade sur le boulevard de Sébastopol », reprises dans le contrat de vente du terrain nu du futur $n^{\circ} 2$, Arch. nat., MC, ET/LI/1441.

3. Le Moniteur universel, 15 juillet 1856, p. 1.

4. Georges-Eugène Haussmann, Mémoires, [réed.] Paris, Le Seuil, 2000, p. 1104.

5. F. Camus, Le Journal des débats, 21 avril 1858.

6. François Lacour, «Fontaine Saint-Michel», Le Monde illustré, 8 mai 1858, Paris, Librairie nouvelle, 1858, t. II, p. 295.

7. Paris, Archives des musées nationaux, K/23.

8. Bibliothèque administrative de la ville de Paris, fonds Davioud, SP 413 b (12) CR Da 2054, dessin daté du 6 avril 1858.

9. Archives de Paris, $1304 \mathrm{~W} / 25 / 2$.

10. Ibid.

11. François Lacour, « Fontaine Saint-Michel », op. cit., p. 295.

12. Tourettes, Patrie, 20 avril 1858.

13. F. Camus, Le Journal des débats, 21 avril 1858.

14. Tourettes, Patrie, 20 avril 1858.

15. Napoléon III, « Discours du 19 octobre 1852 prononcé à Bordeaux », La politique exposée par les discours et proclamations de l'empereur Napoléon III depuis le 10 décembre 1848 jusqu'en février 1868 , Paris, Plon, 1868, $448 \mathrm{p}$.

16. François Lacour, « Fontaine Saint-Michel », op. cit., p. 295.

17. Dominique Jarrassé, «Les fontaines et le décor urbain », Gabriel Davioud, architecte: 1824-1881, cat. expos., Paris Mairies des XVI ${ }^{\mathrm{e}}$ et XIX' arrondissements, 1981-1982, Paris, Délégation à l'Action artistique de la ville de Paris, 1981, p. 46.

18. F. Camus, Le Journal des débats, 21 avril 1858.

19. Adolphe Lance, «La Fontaine Saint-Michel», Encyclopédie d'architecture, Victor Calliat et Adolphe Lance, $\mathrm{n}^{\circ}$ 11, novembre 1860, Paris, Bance, 1860, t. X, p. 163.

20. Dominique Jarrassé, «La fontaine Saint-Michel. Le classicisme controversé », Archives d'architecture moderne, $\mathrm{n}^{\circ} 22,1982$, p. 83.

21. Archives de Paris, $1304 \mathrm{~W} / 25 / 2$.

22. Archives de Paris, D.1Z/78/Place Saint-Michel, p. 1598.

23. Charles Friès, Le Moniteur universel, 23 septembre 1859, p. 1095.

24. Actualités de la Bibliothèque historique de la Ville de Paris, dossier topographique 35, sn, 2 mai 1859.

25. Le Moniteur, 11 avril 1860, p. 411.

26. Le Moniteur, 1er juin 1860, p. 642.

27. Le Moniteur, 16 juillet 1860 , p. 847.

28. Archives de Paris, $1304 \mathrm{~W} / 25 / 2$.

29. Le Moniteur, 13 août 1860, p. 979.

30. Le Moniteur, 14 août 1860, p. 986.

31. Le Moniteur, 17 août 1860, p. 989.

32. Augustin Joseph du Pays, « Fontaine Saint-Michel », L'Illustration, 18 août 1860, p. 110.

33. Georges-Eugène Haussmann, Mémoires, op. cit., p. 1104.

34. Alfred Darcel, «La fontaine Saint-Michel », Gazette des beaux-arts. Courrier européen de l'art et de la curiosité, octobre 1860, tome VIII, p. 50.

35. Charles Blanc, "La fontaine Saint-Michel ", Gazette des beaux-arts. Courrier européen de l'art et de la curiosité, octobre 1860, tome VIII, p. 52.

36. Dominique Jarrassé, « Les fontaines et le décor urbain », op.cit, p. 46. 
37. Jules-Antoine Castagnary, «La Fontaine Saint-Michel », Paris qui s'en va et Paris qui vient, Paris, Alfred Cadart, 1860, repris dans Les Libres Propos, Paris, A. Lacroix, Verbokhoben et $C^{\text {ie }}, 1864$, p. 36.

38. Ibid., p. 29.

39. Thomas von Joest, Claudine de Vaulchier, "Davioud et les places haussmanniennes » Monuments historiques, $\mathrm{n}^{\circ}$ 120, mars-avril 1982, p. 67.

40. Charles Friès, Le Moniteur universel, 23 septembre 1859, p. 1095.

41. Charles Blanc, « La fontaine Saint-Michel », op. cit., p. 52.

42. Jules-Antoine Castagnary, « La Fontaine Saint-Michel », op. cit., p. 35.

43. Lettre du 5 septembre 1870. Archives de Paris, $1304 \mathrm{~W} / 46 / 3$.

44. Archives de Paris, V.M92/1/Fontaines monumentales. Affaires diverses.

45. 35,42 mètres au dessus du niveau de la mer au débouché du pont ; 35,26 rue Saint-Séverin. Archives de Paris, V.011/3359/Nivellements, avant-projet, profil en long du boulevard de Sébastopol, 15 janvier 1859.

46. Alfred Darcel, « La fontaine Saint-Michel », op. cit., p. 47.

47. Victor Fournel, Paris nouveau et Paris futur, Paris, Librairie Jacques Lecoffre, 1868, p. 166 sq.

48. Inventaire général, 1878, p. 122.

49. Victor Fournel, Paris nouveau et Paris futur, op. cit., p. 166.

50. Denis-Louis Destors, « Notice sur la vie et les œuvres de Gabriel Davioud [...] lue au congrès, séance du 14 juin 1881 ", Congrès annuel des architectes à l'École des beaux-arts, IX ${ }^{\mathrm{e}}$ session, Bulletin de la société centrale des architectes, Paris, Ducher, 1881, p. 239.

51. Dominique Jarrassé, « Les fontaines et le décor urbain », op. cit., p. 45.

52. Charles Rossigneux, Discours prononcé à la Société d'encouragement pour l'industrie nationale sur la vie et les œuvres de Gabriel Davioud, le 22 décembre 1882, Paris, Librairie Hachette et cie, 1883, p. 9.

53. Charles Friès, « La fontaine Saint-Michel », op. cit.

54. Paris nouveau illustré, 1868, p. 131.

55. Augustin Joseph du Pays, « Fontaine Saint-Michel », op. cit., p. 110.

56. Ibid.

57. Louis Lazare, Les Quartiers pauvres de Paris, Paris, Études municipales, publications administratives en vente au bureau de la bibliothèque municipale, 1869, p. 125.

58. Jules-Antoine Castagnary, « La Fontaine Saint-Michel », op.cit., p. 37.

59. Paris nouveau illustré, 1868, p. 131.

60. Félix Narjoux, Paris : monuments élevés par la ville, op. cit., p. 5.

61. Augustin Joseph du Pays, « Fontaine Saint-Michel », op. cit., p. 110.

62. François Loyer, Paris XIX ${ }^{e}$ siècle : l'immeuble et la rue, Paris, Hazan, nouvelle éd., 1994, p. 292.

63. Pierre Pinon, Atlas du Paris haussmannien : la ville en héritage du second empire à nos jours, Paris, Parigramme, 2002, p. 113.

64. Architecte Victor Baltard, 1860.

65. Dominique Jarrassé, « Les fontaines et le décor urbain », op. cit., p. 45.

66. Augustin Joseph du Pays, « Fontaine Saint-Michel », op. cit., p. 110.

67. Dominique Jarrassé, « Les fontaines et le décor urbain », op. cit., p. 45.

68. École nationale supérieure des beaux-arts, vol. 4, Esq 330.bmp.

69. Sylvain Bellenger, et Françoise Hamon dir., Félix Duban, 1798-1870 : les couleurs de l'architecte, Paris, Gallimard-Electa, 1996, 264 p.

70. Jules Bourdais, "Gabriel Davioud, architecte, 1824-1881 ", Encyclopédie d'architecture, revue mensuelle des travaux publics et particuliers, Victor Calliat, Paris, A. Morel, 1881, t. X, p. 36.

71. Citée par Adolphe Lance, « La Fontaine Saint-Michel », op. cit., p. 164.

72. François Lacour, « Fontaine Saint-Michel », op. cit., p. 295.

73. Paris nouveau illustré, 1868, p. 131.

74. Victor Fournel, Paris nouveau et Paris futur, op. cit., p. 166. 
75. Daniel Rabreau, Gabriel Davioud, architecte, op. cit., p. 13.

76. Le Moniteur, 3 août 1863, p. 1024.

77. Dominique Jarrassé, « La fontaine Saint-Michel », op. cit., p. 85.

78. Dominique Jarrassé, « Les fontaines et le décor urbain », op. cit., p. 46.

79. Béatrice Lamoitier, dans Daniel Rabreau, Dominique Massounie et Pauline PrévostMarcilhacy dir., Paris et ses fontaines de la Renaissance à nos jours, Paris, Action artistique de la ville de Paris, 1995, p. 184.

80. Inventaire général, 1878, p. 123.

81. Augustin Joseph du Pays, « Fontaine Saint-Michel », op. cit., p. 110.

82. Ibid.

83. Notice officielle citée par Adolphe Joanne, Paris illustré en 1870 : guide de l'étranger et du Parisien, Paris, Hachette, « coll. des guides Joanne », 1870, p. 144.

84. Dominique Jarrassé, « Les fontaines et le décor urbain », op. cit., p. 46.

85. Notice officielle citée par Adolphe Lance, « Fontaine Saint-Michel », op. cit., p. 165.

86. Charles Blanc, «Fontaine Saint-Michel », op. cit., p. 54.

87. Le Moniteur, 13 janvier 1861, p. 58.

88. Le Moniteur, 3 mars 1861, p. 286.

89. Adolphe Joanne, Paris illustré, op. cit., p. 144.

90. Victor Fournel, Paris nouveau et Paris futur, op. cit., p. 166.

91. Le Temps, 16 mai 1893, sn. Actualités de la Bibliothèque historique de la Ville de Paris, dossier topographique 36.

92. Ainsi se lamente la naïade de la fontaine Médicis menacée de destruction dans Arkelio, 1861, p. 6.

93. Jules-Antoine Castagnary, « La fontaine Saint-Michel », op. cit., p. 40.

94. Adolphe Lance, « La fontaine Saint-Michel », op. cit., p. 166.

95. Dominique Jarrassé, « La fontaine Saint-Michel », op. cit., p. 87.

96. Alfred Darcel, « La fontaine Saint-Michel », op. cit., p. 51.

97. Louis Lazare, Les Quartiers pauvres de Paris op. cit., p. 125.

98. Victor Fournel, Paris nouveau et Paris futur, op. cit., p. 166.

99. François Loyer, L'Immeuble et la rue, op. cit., p. 238.

100. Recueil des actes administratifs de la préfecture du département de la Seine, Paris, imprimerie et librairie administrative de Paul Dupont, 1855, p. 167 sqq.

101. Arch. nat., MC, ET/LI/1441.

102. Michaël Darin, "Harmonisation difficile ", Les Places de Paris, XIX ${ }^{e}-X X^{e}$ siècles, Géraldine Texier-Rideau, Michaël Darin dir., Paris, Action artistique de la ville de Paris, 2003, p. 228.

103. Françoise Boudon, «Le regard du XIX ${ }^{\mathrm{e}}$ siècle sur le XVI siècle français : ce qu'on vu les revues d'architectures ", Revue de l'art, 1990, n 1, p. 39-56.

104. Françoise Boudon, « La «maison à loyer » de la ville haussmannienne », Revue de l'art, 1988, vol. $79, \mathrm{n}^{\circ} 1$, p. 69.

105. François Loyer, L'Immeuble et la rue, op. cit., p. 296. 


\section{RÉSUMÉS}

À partir de 1855, Gabriel Davioud entreprend de résoudre avec pragmatisme les difficultés que la crise urbaine accumule au débouché du pont Saint-Michel. Réponse aux entraves à la circulation et lotissement spéculatif d'envergure locale, la place Saint-Michel marque de façon monumentale l'entrée du boulevard de Sébastopol sur la rive gauche. Bordée d'immeubles identiques, ornée d'une belle fontaine, elle s'inscrit à la suite des embellissements du XVIII ${ }^{\mathrm{e}}$ siècle. Toutefois, les exigences circulatoires nouvelles de l'âge industriel bouleversent les rapports traditionnels entre monument et bâti vernaculaire. Objet scénographique et décoratif répondant aux nécessités de la visée, la fontaine Saint-Michel affirme la prise de contrôle de l'espace urbain par le régime issu du coup d'État du 2 décembre 1851. Les fonctions politiques qu'elle remplit à l'évidence retiendront ici d'abord l'attention. On verra ensuite combien les formes nouvelles de monumentalité qu'elle met en œuvre font débat. On s'attardera enfin à préciser les rapports hiérarchiques inédits entre bâti vernaculaire et architecture d'exception qu'inaugure la place Saint-Michel.

As from 1855, architect Gabriel Davioud decides to resolve in a pragmatic manner the difficulties of the urban crisis that came to a head at the mouth of the bridge St Michel. The St Michel square provides the solution to traffic problems, represents a local division of land for building and is the main entrance of the Boulevard Sébastopol on the left bank. Surrounded by identical buildings and adorned by a beautiful fountain, the square represents the continuation of the embellishments of the 18th Century. However, the traffic requirements of the industrial era completely overhaul the relationship between the monuments and plain buildings that surrounds it. The fountain is a stenographic and decorative object that reflects how the Imperial regime wished to control urban space. The political role it plays should be taken first into consideration. Then the debate regarding the exceptional features of the fountain is analysed in depth. The new hierarchical forms between monuments and plain buildings built around launched with the St Michel square are discussed at a later stage.

$\mathrm{Ab} 1855$ versuchte Gabriel Davioud die Folgen der krisenhaften Stadtentwicklung pragmatisch zu beseitigen, die besonders konzentriert an der Einmündung zu der Brücke Saint-Michel waren. Die Errichtung des Platzes Saint-Michel gestattete einerseits die Lösung der Verkehrsstörungen, andererseits die Bebauung von bedarfsmäßigen Mietshäusern. Diese verlieh dem Boulevard Sébastopol einen eindrucksvollen Übergang zum linken Ufer der Seine. Der Platz, der von homogenen Wohngebäuden umrandet und mit einem schönen Brunnen ausgestattet wurde, passte sich zwar gut an die Verschönerungen des 18. Jahrhunderts an. Die traditionellen Verhältnisse zwischen Pracht- und Allgemeinbauten wurden indessen infolge der neuen Verkehrsanforderungen gemäß der damaligen industriellen Entwicklung völlig verändert. Der Bau des Brunnens Saint-Michel, der zwar nach perspektivischen und ästhetischen Gesetzen eingerichtet ist, entspricht auch dem stärkeren Willen des aus dem Staatsstreich vom 2. Dezember 1851 entstandenen Regimes, den urbanen Raum zu bewältigen. Der Aufsatz nimmt sich erstens vor, die augenscheinliche politische Aufgabe dieser Einrichtung zu überprüfen. Zweitens untersucht er die neuen monumentalen Bauformen des Brunnens, die damals umstritten waren. 
Zum Schluss wendet er sich an die ganz neuen Verhältnisse zwischen Pracht- und Allgemeinbau, die am Platz Saint-Michel eingeführt wurden.

\section{AUTEUR}

\section{GRÉGOIRE ALESSANDRI}

Grégoire Alessandri, né en 1964, est actuellement correspondant législatif à la Commission européenne. Il a entamé, de nouvelles études en 2006 à l'École du Louvre. Après un premier cycle, il a présenté un mémoire de M1 intitulé La place Saint-Michel à Paris : un exemple de composition urbaine hiérarchisée Dir. de Catherine Chevillot et Alice Thomine-Berrada. Aujourd'hui en M2, il travaille Dir. d'Alice Thomine-Berrada et de Dominique Jarrassé à la préparation d'un mémoire consacré aux huit églises construites à Paris sous le directorat de Victor Baltard au service des Travaux de la Ville (1860-1870). 